\title{
LA ALIANZA TERAPÉUTICA EN LA DANZA MOVIMIENTO TERAPIA CON PACIENTES CON DISCAPACIDAD INTELECTUAL. Estudio de caso
}

\section{THERAPEUTIC ALLIANCE IN DANCE/MOVEMENT THERAPY WITH PATIENTS WITH INTELLECTUAL DISABILITY. Case study}

\author{
Silvia Barnet-Lopez ${ }^{*}$, Meritxell Pacheco', Heidrun Panhofer ${ }^{2}$, \\ Peter Zelaskowski ${ }^{2}$, Susana Pérez-Testor ${ }^{1,2}$ y Myriam Guerra-Balic ${ }^{1}$ \\ ${ }^{1}$ Facultat de Psicologia, Ciències de l'Educació i de l'Esport (FPCEE) - Blanquerna. \\ Universitat Ramon Lull. \\ ${ }^{2}$ Universitat Autónoma de Barcelona.
}

Cómo referenciar este artículo/How to reference this article:

Barnet-Lopez, S., Pacheco, M., Panhofer, H., Zelaskowski, P., Pérez-Testor, S. y Guerra-Balic, M. (2016). La alianza terapéutica en la danza movimiento terapia con pacientes con discapacidad intelectual. Estudio de caso. Revista de Psicoterapia, 27(104), 233-251.

\begin{abstract}
Resumen
Presentamos un estudio de caso cualitativo descriptivo. La participante es una mujer de 39 años con Discapacidad Intelectual, especificamente Síndrome de Down, que realizó una intervención individual en Danza Movimiento Terapia (DMT). Los objetivos del estudio son: (1) conocer los elementos relevantes de la Alianza Terapéutica (AT) que se estableció durante la intervención consistente en 19 sesiones en DMT y (2) observar si estos elementos de la AT contribuyen al éxito de los objetivos de la intervención. Los objetivos de la intervención fueron: aumentar el conocimiento del propio cuerpo, aumentar el control de impulsos y la tolerancia a la frustración, mejorar la comunicación y la relación con el otro y fomentar la iniciativa, la creatividad, la seguridad y su autonomía. El terapeuta realizó notas de campo para recoger los elementos más importantes del caso en relación a la construcción de la AT y de los objetivos de la intervención. Posteriormente se realizó un análisis de contenido. Los resultados muestran que se construyó una AT saludable, lo cual permitió alcanzar los objetivos de la terapia. Así mimo, este estudio de caso es una aportación al ámbito de la DMT, que nos permite conocer mejor el trabajo realizado en este tipo de intervenciones.
\end{abstract}

Palabras clave: Relación terapéutica, Alianza terapéutica, Discapacidad intelectual, Danza Movimiento Terapia.

Fecha de recepción: 1/03/2016. Fecha de aceptación v1: 15/04/2016. Fecha de aceptación v2: 29/05/2016 Correspondencia sobre este artículo:

E-mail: SilviaBL0@blanquerna.url.edu

Dirección postal: Silvia Barnet-Lopez. c/Cister 34, 08022 Barcelona, España

(C) 2016 Revista de Psicoterapia 


\begin{abstract}
This is a qualitative descriptive case study. The participant was a woman of 39 years with Intellectual Disability, specifically Down syndrome, who performed an individual intervention in Dance/Movement Therapy (DMT). The objectives of this study were: (1) to know the relevant elements of the Therapeutic Alliance (TA) which was established during the therapeutic process for 19 sessions DMT and (2) to observe if these elements of the TA contributed to the success of the objectives of the intervention. The objectives of the intervention were: to increase knowledge of her own body, to increase impulse control and frustration tolerance, to improve communication and relationship with the others and encourage initiative, creativity, security and autonomy. The therapist got field notes to collect the most important elements of the case in relation to the construction of the AT and objectives of the intervention. Later, it was performed a content analysis. The results showed that a healthy AT was constructed, which allowed achieving the objectives of the therapy. Additionally, this case study becomes contribution to the field of DMT and it allows us to better understand the work developed in these interventions.
\end{abstract}

Keywords: Therapeutic relationship, Therapeutic alliance, Intellectual disability, Dance Movement Therapy. 


\section{INTRODUCCIÓN}

Presentamos el estudio de un caso en Danza Movimiento Terapia (DMT) en una paciente con Discapacidad Intelectual (DI). Este trabajo se enfoca en conocer los elementos relevantes de la Alianza Terapéutica (AT) que se estableció durante el proceso terapéutico durante 19 sesiones.

\section{Danza Movimiento Terapia}

La DMT se encuentra dentro de las terapias creativas, como la arteterapia o la musicoterapia, las cuáles han demostrado su eficacia en la contribución de cambios de nivel psicológico y relacional sobretodo en personas que presentan dificultades a la hora de expresar o de elaborar conflictos personales (Rodas, 2015). A través de algunas de las terapias creativas se ha utilizado el movimiento del cuerpo y los gestos en conjunto con la creatividad y la espontaneidad de los sujetos, como medios para llevar a cabo una intervención (Arregui, 2010).

La DMT es definida como "una especialidad en psicoterapia que utiliza el cuerpo y sus expresiones no verbales como un proceso para promover la integración emocional, cognitiva y física del individuo" (Panhofer, 2005, p.50). Está presente una interacción contante entre el cuerpo y la mente (Vella y Torres, 2012).

Se considera el movimiento como un medio que utiliza la DMT para intervenir y provocar el cambio psicológico en las personas en todas sus áreas (cognitivas, emocionales, físicas y relacionales) ayudado por la danza y también el lenguaje (Rodas, 2015). Es una herramienta de expresión y comunicación que permite transmitir emociones, sentimientos, pensamientos y vivencias (Ballesta, Vizcaíno y Mesas, 2011; Laban, 1978). Fischman (2005) afirma que la atención en la conciencia de los estados corporales viene de las variaciones del movimiento o acción corporal. Todo ello contribuye a la reconexión o al descubrimiento de estados corporales internos, sensaciones, emociones y sentimientos. Así pues, permite una expresión más libre del sujeto a través de elementos creativos, sin forzar un razonamiento previo, favoreciendo una conexión terapeuta-paciente más próxima (Hurley, Tomasulo y Fadt, 1998).

Borja y Solís (2012) proponen la intervención a través de la danza y la música para mejorar la comunicación y aumentar el conocimiento del propio cuerpo en personas con DI.

Otros estudios también ponen de manifiesto las aportaciones de la DMT en población con DI a nivel emocional y de esquema y conocimiento corporal (BarnetLópez, Pérez-Testor, Cabedo-Sanromà, Gozzoli, Oviedo, y Guerra-Balic, 2015; Levy, 1988). A pesar de observar un aumento en las investigaciones en este ámbito, la DMT es una de las terapias creativas menos conocida y aplicada en estudios científicos por ello es importante incrementar las evidencias científicas en este campo (Koch, S., Kunz, T., Lykou, S., y Cruz, R., 2014).

Una de las pioneras de la DMT, Marian Chace, afirma que la DMT en DI será importante que el terapeuta tenga un estilo directivo y muy activo, interactuando 
con la persona en movimiento y verbalmente. Destacamos la técnica de la repetición como una herramienta eficaz de trabajo y la técnica de espejar, donde el terapeuta logra una conexión con el paciente a nivel emocional; pudiendo comprenderlo y aceptarlo en el punto en que este se encuentra, ofreciendo de esta manera al paciente una escucha que permite validar la experiencia del mismo (Chace, 1953; Levy, 1992,1988). Se pueden utilizar distintos materiales como cuerdas, telas o pelotas, orientados a ampliar el vocabulario de movimiento y que también facilitarán la comunicación de la persona, su interacción con el grupo, la exploración a través del juego y la relación terapéutica (Panhofer, 2005).

La relación del terapeuta y del paciente en la DMT involucra relevantes elementos como el contacto físico y la proximidad ya que se crea un dialogo entre el cuerpo del paciente y el del terapeuta (Mac Donald, 1992). Así pues, se considera que una "psicología de dos personas, es en realidad, una psicología de dos cuerpos, y no se trata sólo de un encuentro de mentes sino de persones que incluyen cuerpos" (Aron, 1999, p. 267). El encuentro terapéutico permite una nueva forma de relación donde paciente y terapeuta construyen una manera propia de interactuar y de comunicarse (Sassenfeld, 2008).

\section{Discapacidad Intelectual}

Según el DSM-V la DI es un trastorno del neurodesarrollo que incluye tres criterios básicos: (1) Déficits en el funcionamiento intelectual, (2) déficits en el funcionamiento adaptativo, (3) inicio en el período de desarrollo. Según el nivel de afectación en las áreas: conceptual, social y práctica, se establecen los siguientes niveles de gravedad: Leve, Moderado, Grave, Profundo (AAIDD, 2010; American Psychiatric Association. 2013).

Según la Asociación Americana de Discapacidades Intelectuales y del Desarrollo, Existen diversas causas de DI, como las cromosomopatías, donde estará incluido el Síndrome de Down (AAIDD, 2010).

\section{Alianza Terapéutica}

Se considera que la psicoterapia no se podría concebir sin el establecimiento de una relación entre paciente y terapeuta (Corbella, Balmaña, Fernández-Álvarez, et al., 2009). Uno de los elementos más relevantes de la relación terapéutica es la Alianza Terapéutica (AT) considerada como uno de los mejores predictores de eficacia de los resultados en psicoterapia (Andrade, 2005; Horvath y Bedi, 2002). Corbella y Botella (2003) en su estudio refieren que la importancia que se ha atribuido a la AT en las investigaciones pone de manifiesto la importancia de la dimensión relacional entre terapeuta y paciente. Se considera que la AT será un factor facilitador para el desarrollo del proceso terapéutico, y por lo tanto mejorará la sintomatología de la persona (Botella y Corbella, 2011).

El concepto de AT ha ido evolucionando (Corbella y Botella, 2002). Greenson (1967) nombró por primera vez este concepto. Posteriormente Bordin (1979) 
desarrolló y conceptualizó los tres elementos que la componen: acuerdo de las tareas, acuerdo en las metas y vínculo positivo. Debe existir un acuerdo entre pacientes y terapeuta para poder trabajar con el mismo objetivo, y las dos partes tienen un papel activo en la terapia (Andrade, 2005).

Se han realizado diversas investigaciones en relación a la importancia de la AT y los elementos que la componen (Etchevers, Garay, Korman, González, y Simkin, 2012). Horvath (1981) demostró que existía correlación entre la empatía del terapeuta percibida por el paciente, y por otro lado algunos aspectos relacionados con la alianza. Peschken y Johnson (1997) afirmaron que las "actitudes facilitadoras" constituyen la construcción de la AT. Además demostraron la correlación positiva entre la confianza depositada por el terapeuta y la condiciones facilitadoras, correlacionando el segundo elemento con la confianza del paciente.

Beck, Rush, Shaw y Emery (1983) hablan de la confianza básica como un elemento que se fomenta a través de la empatía, aceptación y autenticidad. La definen como una percepción del paciente hacia la relación que establece con el terapeuta, la cual considera buena y segura, pudiendo expresarse con más libertad con el objetivo de encontrar una solución. La colaboración terapéutica de las dos partes es esencial para poder trabajar en equipo.

Watson y Geller (2005) consideran que los terapeutas que muestran empatía, congruencia y aceptación por sus pacientes podrán desarrollar una mejor negociación de los objetivos terapéuticos, y por lo tanto una construcción más positiva del vínculo entre ambos.

En el caso que presentamos deberemos tener presentes posibles dificultades relacionales que afectarán a la AT. La relación terapéutica se ve influenciada por algunos factores previos e independientes de la terapia, como variables del paciente en relación a la severidad del cuadro clínico, o sus conductas de apego en sus primeros años, así como sus habilidades adquiridas a nivel relacional, o sus experiencias o actitudes previas (González, 2005). Por lo tanto, en este caso las limitaciones cognitivas, relacionales y de lenguaje serán elementos a tener muy presentes durante la creación de la AT. En DI estamos trabajando con un tipo de población donde encontraremos personas y/o grupos muy heterogéneos, ya que existe un amplio abanico de niveles de funcionamiento, de discapacidades y de cualidades (Jané, 2004).

\section{OBJETIVO}

Los objetivos principales son: (1) identificar los elementos relevantes de la Alianza Terapéutica que aparecen a lo largo de una intervención en Danza Movimiento Terapia en un caso con Discapacidad Intelectual y (2) observar si estos elementos de la Alianza Terapéutica contribuyen al éxito de los objetivos de la intervención.

Los objetivos específicos son: (1) describir el proceso terapéutico de una intervención en Danza Movimiento Terapia con Discapacidad Intelectual y (2) 
observar la evolución de los objetivos de la intervención a lo largo del proceso terapéutico en un caso de Danza Movimiento Terapia con Discapacidad Intelectual.

\section{MÉTODO}

Se trata de un estudio cualitativo, concretamente un estudio de caso descriptivo. Definimos el estudio de caso como "la examinación o investigación de forma intensiva, detallada y profunda de una unidad simple: el caso" (Luck, Javkdon y Usher, 2006, p. 104).

La selección del caso fue por conveniencia (Miles y Huberman,1994). Tal y como afirma Starman (2013) nos encontramos delante de un estudio diacrónico, ya que se sigue un caso a lo largo de todo el proceso psicoterapéutico, analizando y observando los cambios que se producen en el paciente como resultado de la intervención.

Este estudio ha sido respetuoso con los principios éticos que rigen cualquier investigación realizada con seres humanos de acuerdo a la declaración de Helsinki (Asociación Médica Mundial, 1964). Se ha preservado el anonimato del paciente y del centro.

\section{Descripción del participante}

Mujer de 39 años a la que llamaremos Lucía. Lucía está diagnosticada de Síndrome de Down. No toma medicación. Vive con sus padres. Tiene dos hermanos mayores que viven en un país extranjero. Le gusta escuchar música, ver la televisión y practicar aerobic. Los educadores y la psicóloga del centro de terapia ocupacional destacan: (1) Dificultades en la relación con el otro. (2) Falta de tolerancia a la frustración: "sólo hace lo que ella quiere". (3) Rigidez corporal: "esta en constante tensión".

Previamente a la intervención el terapeuta realizó observaciones de Lucía en el taller ocupacional. Presentamos un resumen de sus consideraciones:

- A nivel corporal: cuerpo rígido con postura tensa, destaca su nivel de energía inconstante y su dificultad para estar quieta. Su repertorio de movimiento es reducido y destacan patrones repetitivos de movimiento: frotar sus manos, tocarse el pelo y mirarse su mano izquierda.

- A nivel relación: "suele mirar hacia abajo cuando no le gustaba algo", "su rostro es muy expresivo cuando no está de acuerdo con algo", aunque permite la proximidad del otro pero solamente cuando necesita alguna cosa; presenta muchas limitaciones en el lenguaje oral y permite el contacto visual y físico, si bien solamente de las personas que ella escoge.

A partir de las necesidades detectadas en Lucía, se decidió trabajar con ella mediante DMT, puesto que este tipo de terapia facilita la expresión emocional, fomenta la conexión entre el cuerpo y la mente y, además, propicia la ampliación de registros de movimientos proporcionando un mayor conocimiento del propio cuerpo (Fischman, 2005). 


\section{Instrumento}

El terapeuta realizó notas de campo (Gibbs, 2007), anotaciones no continuadas, durante y después de cada una de las sesiones de la intervención, para recoger los elementos más importantes del caso en relación a la construcción de la AT y de los objetivos de la intervención.

También se realizaron supervisiones del caso con un terapeuta experto en estudios de caso dentro de la disciplina de la DMT.

\section{Análisis de los datos}

Se realizó un análisis de contenido (Ruiz, 1996) para analizar las notas de campo. Concretamente se utilizó un método deductivo.

En primer lugar se determinaron los elementos relevantes de la AT según las referencias consultadas, diferenciando entre aquellos elementos que ayudaron al vínculo (P) y aquellos que lo dificultaron (N) (Bordin 1979,1994; González, 2005; Horvath y Greenberg, 1989; Rogers y Resenberg, 1981; Winkler, Cáceres, Fernández y Sanhueza, 1989).

Así pues, se obtuvieron diferentes categorías para analizar la construcción de la AT y evolución de los objetivos trabajados en la intervención (ver tabla 1).

\section{Tabla 1}

\section{Tabla de categorías}

\begin{tabular}{ll}
\hline Elementos relacionados & - capacidad de tolerar cierto grado de frustración \\
con la AT & - confianza básica y/o aceptación \\
(P o N) & - identificación con las metas de la intervención \\
& - metas y objetivos comunes \\
& - capacidad de vincularse y/o empatía \\
& - actitud de interés \\
& $-\quad$ integración de elementos del encuadre, \\
& - permitir o iniciar contacto \\
& $-\quad$ validar la experiencia del paciente y autenticidad \\
& (congruencia y coherencia). \\
\hline En la sesión & elementos destacados de la sesión en relación a las \\
& actividades propuestas para trabajar los objetivos de la \\
intervención.
\end{tabular}

Posteriormente procedimos a la lectura del diario de campo y se identificaron las categorías mencionadas en la Tabla 1. Luego el mismo experto supervisor del caso realizó la revisión de los resultados obtenidos, estos no se consideraron 
definitivos hasta lograr un acuerdo interjueces en cada una de las categorías.

\section{Intervención}

La intervención se realizó en el contexto de un taller ocupacional en el que Lucía asistía diariamente. Se planificaron 19 sesiones individuales de DMT de 60 minutos cada una y desarrolladas con periodicidad semanal. Las sesiones se estructuraron según el Modelo de Chace (1953):

1. Bienvenida.

2. Calentamiento: Preparamos el cuerpo para el movimiento:

- Movimientos enfocados a elevar la temperatura corporal, como la marcha rítmica.

- Movilización de grandes articulaciones del cuerpo, con movimientos como rotaciones o flexiones, y siguiendo un orden que favorezca el movimiento de todas las partes del cuerpo.

- Un calentamiento específico centrado solamente en aquellos músculos y articulaciones que se utilizaran más en la sesión.

3. Transición-Proceso: Trabajamos con la paciente dentro de un proceso nodirectivo a través de la creatividad, su simbolización, juegos y/o bailes.

4. Relajación: Se realiza si el terapeuta lo considera necesario para la paciente.

5. Cierre: Momento de vuelta a la calma. Un espacio de contención, de intercambio, de reflexión y de integración de las temáticas emergentes en el proceso.

Se formularon los siguientes objetivos de intervención:

1. Aumentar el conocimiento del propio cuerpo.

2. Aumentar el control de impulsos y la tolerancia a la frustración.

3. Mejorar la comunicación y la relación con el otro.

4. Fomentar la iniciativa, la creatividad, la seguridad y su autonomía.

Estos objetivos fueron empleados para plantear los contenidos principales de la intervención, teniendo presentes las limitaciones de Lucía.

Se proporcionó a Lucía un espacio contenido para aumentar su seguridad y también se propusieron diferentes rutinas o rituales. Se exploraron diferentes maneras de entrar en contacto en la relación; se puso el foco en cómo cuidar el espacio y en clarificar los elementos que fueron configurando el encuadre. Se reforzó a Lucía positivamente lo cuál permitió que fuera cogiendo más iniciativa. Se utilizó la música herramienta facilitadora en la relación, ya que Lucía mostraba mucho interés por algunas canciones, y también como una herramienta de apoyo tanto a nivel de estímulo como de estructura.

\section{RESULTADOS}

A continuación realizaremos un recorrido por el proceso terapéutico en el que observaremos los diferentes "momentos de encuentro" (Stern et al., 1998, p. 5) entre 
Lucía y el terapeuta vinculados a la AT, y destacaremos los momentos de cambio asociados (para mayor información ver apéndice).

\section{Primeras sesiones: Inicio del proceso}

Estaban muy presentes las conductas obsesivas y de negación (ver apéndice Tabla 2 y 3 ), pero durante el proceso Lucía pudo explorar otras alternativas y tolerar mejor la frustración. Referente a la AT destacamos que, a pesar de que la primera sesión se centra en establecer el vínculo terapéutico saludable, el terapeuta parece ser invisible para Lucía. Además, como se aprecia en la Tabla 2, Lucía presenta dificultades en la integración de elementos del encuadre (ver apéndice Tabla 2).

En las sesiones dos y tres Lucía parece estar más presente en lo que sucede en su entorno y empieza a reconocer el espacio, elementos que contribuyen a una buena AT. También muestra movimientos repetitivos que parecen darle seguridad, como el balanceo y los giros. Al final de las sesiones, se propone un espacio de relajación donde se realiza un pequeño masaje de pies que permite observar que Lucía muestra un pensamiento muy concreto: para ella el masaje es para los calcetines y no parece comprender que detrás de ellos están sus pies (ver apéndice Tabla 2).

Destacan sus movimientos repetitivos, además de un registro de movimiento muy limitado. Se empezará a trabajar desde el punto en que ella se encuentra, teniendo presente sus limitaciones y puntos fuertes (ver apéndice Tabla 2 y Tabla 3 ).

\section{Desarrollo del proceso}

Lucía empieza a integrar los elementos del encuadre y a establecer rituales, al inicio y al final de la sesión. Se observan más momentos de interacción entre terapeuta y paciente. Lucía en algunos momentos se dirige al terapeuta; al final de la sesión esperándola para salir del aula, le dice "vamos" y señala la puerta de salida (ver apéndice Tabla 4).

Se observan elementos que permiten ir avanzando en el proceso y que facilitan la buena AT, incrementando los intercambios entre paciente y terapeuta. Referente a los objetivos de la intervención se pueden ver algunos cambios a nivel corporal. El cuerpo de Lucía en algunos momentos parece menos rígido y permite la introducción de pequeños cambios en su movimiento a partir de sus movimientos repetitivos como son los giros. Lucía empieza a compartir y a hacer más participe a el terapeuta de lo que sucede (ver apéndice Tabla 5). Se ve a Lucía más segura y empieza a coger más autonomía queriendo hacer cosas por ella misma, se atreve a proponer movimientos para el calentamiento, parecidos a los trabajados en anteriores sesiones (ver apéndice Tabla 6).

Con las propuestas en movimiento puede experimentar otro tipo de movimientos, como por ejemplo un flujo más libre. También observamos un cambio en el espacio. Lucía utiliza toda la sala, tanto el espacio directo (centrándose en un foco de atención), como el espacio indirecto (utilizando el espacio de forma más flexible pasando por todas las partes de la sala e incluso en algún momento desorientándose). 
Este momento del proceso destacamos: el hecho de compartir en la sesión, aceptar propuestas y poder trabajar en la misma dirección terapeuta y paciente (ver apéndice Tabla 6).

En la sesión 12 Lucía coge la caja de los colores de manera brusca, la caja se rompe y los colores caen. Delante de una situación que no esperaba se queda bloqueada sin saber cómo reaccionar y parece que no presta atención a las palabras del terapeuta. El terapeuta pone palabras a lo sucedido, pero no se observa ninguna reacción, Lucía mira al suelo y su mirada parece perdida. Posteriormente, la sesión continúa y ella parece no estar presente. La situación recuerda a una de las sesiones iniciales. Al final de la sesión Lucía parece que puede conectar con lo sucedido y explica que uno de los educadores se enfadó con ella por una situación parecida (ver apéndice Tabla 7).

En los siguientes encuentros Lucía sigue teniendo una actitud activa y con una mayor iniciativa dentro de la sesión. Lucía vuelve a tirar la caja de colores; esta vez también queda unos instantes bloqueada pero puede escuchar al terapeuta y recogemos los colores con su ayuda. Se la observa tranquila y puede seguir con la sesión estando más presente en esta ocasión (ver apéndice Tabla 8).

La sesión 15 fue como retroceder al inicio. El terapeuta parece invisible y al mismo tiempo destacaba la rigidez corporal y movimientos de giros repetitivos con balanceo (ver apéndice Tabla 9).

En los encuentros posteriores se proponen diferentes opciones para trabajar en movimiento. Se trabajan aspectos como el ritmo (corporal y con instrumentos de percusión) que ayuda a Lucía a crear una estructura e ir cogiendo seguridad, y luego poder ir explorando ritmos nuevos para ella. Además, a través de juegos con ritmos, Lucía puede escuchar los ritmos de el terapeuta y responder a ellos, encontrando nuevas formas de comunicación más allá de la palabra. Lucía descubre una nueva manera de relacionarse (sustentada por un encuadre y unos límites), y un vínculo en el que ella puede ofrecer al otro y disfrutar del aquí y del ahora. Se establece una relación diferente a las relaciones del taller donde Lucía solamente se comunica cuando necesita ayuda (ver apéndice Tabla 10).

\section{Última sesión: El cierre del proceso}

La última sesión fue planteada teniendo presentes los rituales que se habían ido creando en el proceso, y se compartieron canciones y movimientos representativos. El terapeuta puso palabras a lo que iba sucediendo recordando que era el día del cierre. Lucía mostró una actitud más cercana e inició en diferentes momentos movimientos con contacto físico (ver apéndice Tabla 11).

\section{DISCUSIÓN}

Este estudio de caso es un ejemplo del por qué no podemos entender la terapia sin entender la relación que se establece entre paciente y terapeuta. Se observa cómo la AT se crea tanto gracias a la implicación del paciente como del terapeuta 
(Andrade, 2005). Están presentes en este caso algunos de los elementos esenciales para una AT saludable: encuadre claro, espacio seguro, proximidad, confianza, ir en una misma dirección y, sobre todo, observar lo que el paciente trae sesión tras sesión, trabajar en relación a sus necesidades y tolerar su ritmo (Bordin 1979,1994; González, 2005; Horvath y Greenberg, 1989; Rogers y Resenberg, 1981; Winkler, et al., 1989).

En este caso, a pesar de estar presentes limitaciones cognitivas, intelectuales y de lenguaje, el elemento relacional va más allá de ellas. La capacidad para comprender al otro es algo que está en la naturaleza de cada persona independientemente de sus características (Fischman, 2005). Así pues, la AT se fue construyendo y reforzando durante todo el proceso, y esto contribuyó a la consecución de los objetivos de la intervención:

- Aumentar el conocimiento del propio cuerpo: El trabajo del esquema corporal y el explorar nuevas cualidades de movimiento como un flujo corporal más libre, posibilitaron disminuir la rigidez corporal de Lucía (Laban, 1987), además de aumentar el conocimiento de su cuerpo (BarnetLópez, et al., 2015).

- Aumentar el control de impulsos y la tolerancia a la frustración: Durante la sesiones ofrecimos a Lucía alternativas para disminuir sus conductas obsesivas y de negación; ella pudo aceptar bien algunas de las propuestas. Lucía fue descubriendo una nueva manera de relacionarse con el otro, pudo aumentar su tolerancia y disminuir su impulsividad frente a situaciones nuevas y/o que le provocaban frustración.

- Mejorar la comunicación y la relación con el otro: Lucía presentaba muchas limitaciones en la comunicación y la relación. Durante el proceso terapéutico Lucía exploró nuevas maneras de comunicación (Hurley, et al., 1998); se fomentó el lenguaje no verbal con los ritmos, los sonidos y el movimiento.

- Fomentar la iniciativa, creatividad, seguridad y autonomía: El aumento del conocimiento corporal y del registro de movimiento incrementó la seguridad, autonomía e iniciativa de Lucía; observamos a Lucía cada vez más participativa. Además se fomento la creatividad gracias a la utilización de materiales muy diferentes que permitieron a Lucía explorar nuevos movimientos cómo por ejemplo pelotas, telas o diferentes estilos de música (Panhofer, 2005).

Se observa que la creación de la AT fue saludable. Las propuestas de Lucía eran escuchadas y reforzadas, pero también había espacio para escuchar al otro y para tolerar sus intereses o propuestas. Como hemos visto en las primeras sesiones Lucía no buscaba el contacto con el terapeuta y parecía no integrar nada de lo que había a su alrededor. Pero a partir de la sesión cuatro Lucía empezó a integrar elementos del encuadre y a establecer rutinas y rituales en la sesión, como quitarse los zapatos o sentarse para empezar la sesión. 
En las sesiones aparecieron rituales tales como el baile de despedida o como el masaje de pies, también la construcción de un lenguaje propio como el pam, pam que simboliza el calentamiento o la mirada hacia el suelo cuando Lucía quería expresar que algo no le gustaba. Así pues, el terapeuta y Lucía fueron construyendo en sus encuentros su propia manera de interactuar, tal y como sostiene Sassenfeld (2008).

A medida que avanza el proceso, elementos como el contacto físico y la proximidad fomentan la construcción de nuevos diálogos (Mac Donald, 1992). Lucía muestra que tiene la capacidad para vincularse al otro y establecer cierta confianza. En la mitad del proceso vemos a una Lucía que busca la proximidad y aumenta su contacto visual. Se muestra más receptiva a lo que sucede en su entorno y acepta algunas de las propuestas del terapeuta. Referente al contacto físico, se da en muy pocas ocasiones; aparece por primera vez en la sesión diez, cuando al terminar la sesión Lucía se acerca al terapeuta, coge su cara con una mano y después le da un beso de despedida.

Lucía experimentó otro tipo de vínculo diferente al que solía establecer con sus compañeros o educadores del centro. Una comunicación más allá de las demandas de Lucía, donde los intereses del otro también pueden estar presentes. La aplicación de la técnica de espejar (Chace, 1953; Levy, 1992) le permite al terapeuta conectar con las necesidades de movimiento de Lucía y a Lucía empezar a tener presente al terapeuta durante las sesiones. Los momentos de juego compartido fomentaron la comunicación expresiva y comprensiva, y el trabajo con ritmos fue una buena herramienta para trabajar el intercambio comunicativo con Lucía a nivel no verbal. Todo ello en un espacio con unos límites y un encuadre determinado que podía hacer la función contenedora para Lucía. Así mismo el trabajo a través del movimiento, de la expresión no verbal, permitió a Lucía una nueva manera de relación que contribuyó en lograr el éxito en los objetivos terapéuticos, a pesar de sus limitaciones cognitivas y expresivas, coincidiendo con las aportaciones de Rodas (2015).

\section{Limitaciones del estudio y futuras líneas de investigación}

Este trabajo presenta las siguientes limitaciones; la utilización de un solo instrumento (notas de campo) y la imposibilidad de generalizar los resultados, ya que como estudio de caso se centra en la particularidad y en la profundización del caso. Así mismo se proponen para futuras líneas de investigación la realización de un estudio de casos múltiples incorporando otros instrumentos para complementar el análisis, como podría ser un registro de observación.

\section{CONCLUSIONES}

Este estudio es una contribución al ámbito de la DMT. La descripción en profundidad del caso nos permite conocer mejor el trabajo que realiza la DMT en personas con DI.

Los elementos relevantes de la AT que aparecieron a lo largo de la intervención 
fueron: creación de un espacio seguro, integración de los elementos del encuadre, aumento de la confianza, tolerancia del ritmo de Lucía, seguir paciente y terapeuta una misma dirección. Debemos destacar que las limitaciones de Lucía debido a la DI dificultaron la integración del encuadre, por ello consideramos que la repetición de algunos elementos como por ejemplo los rituales de bienvenida y una estructura muy clara y marcada a lo largo de las sesiones son elementos especialmente importantes en este tipo de población.

Se construyó una AT saludable, lo cual permitió alcanzar los objetivos de la terapia: se observa un aumento del esquema corporal y un mayor conocimiento de su propio cuerpo, Lucía aumentó su tolerancia a la frustración en diferentes momentos y mostró un aumento en el control de impulsos, además incrementó la comunicación y aumentó la relación con el terapeuta, finalmente también observamos en el análisis de las sesiones un aumento en la iniciativa, la creatividad, la seguridad y la autonomía a medida que la intervención avanza.

\section{Apéndice}

\section{Tabla 2}

Se inicia el proceso: Sesión 1.

\begin{tabular}{|c|c|c|c|}
\hline Alianza Terapéutica & En Sesión & Su Movimiento & Anotaciones \\
\hline $\begin{array}{l}\text { Falta de tolerancia } \\
\text { frustración }(\mathrm{N})\end{array}$ & Desorientación & $\begin{array}{l}\text { Utiliza solo una } \\
\text { pequeña parte del }\end{array}$ & Soy invisible \\
\hline Clarificación de alaunos & $\begin{array}{l}\text { No acepta propuestas } \\
\text { y/o se cansa muy }\end{array}$ & espacio & $\begin{array}{l}\text { Siento } \\
\text { inseguridad }\end{array}$ \\
\hline elementos del encuadre & rápido de ellas & Destaca el movimiento & \\
\hline $\begin{array}{l}\text { horario, espacio, } \\
\text { estructura sesión (P) }\end{array}$ & Enfado cuando no se & $\begin{array}{l}\text { de balanceo que Lucía } \\
\text { repite durante gran }\end{array}$ & $\begin{array}{l}\text { Frustración de } \\
\text { mis }\end{array}$ \\
\hline No integración de & hace lo que ella quiere & parte de la sesión & $\begin{array}{l}\text { expectativas } \\
\text { iniciales }\end{array}$ \\
\hline $\begin{array}{l}\text { elementos del encuadre } \\
\text { (N) }\end{array}$ & $\begin{array}{l}\text { No reconoce partes } \\
\text { del cuerpo }\end{array}$ & No existe contacto visual & \\
\hline
\end{tabular}




\section{Tabla 3}

Primeras sesiones del proceso: Sesiones 2 y 3.

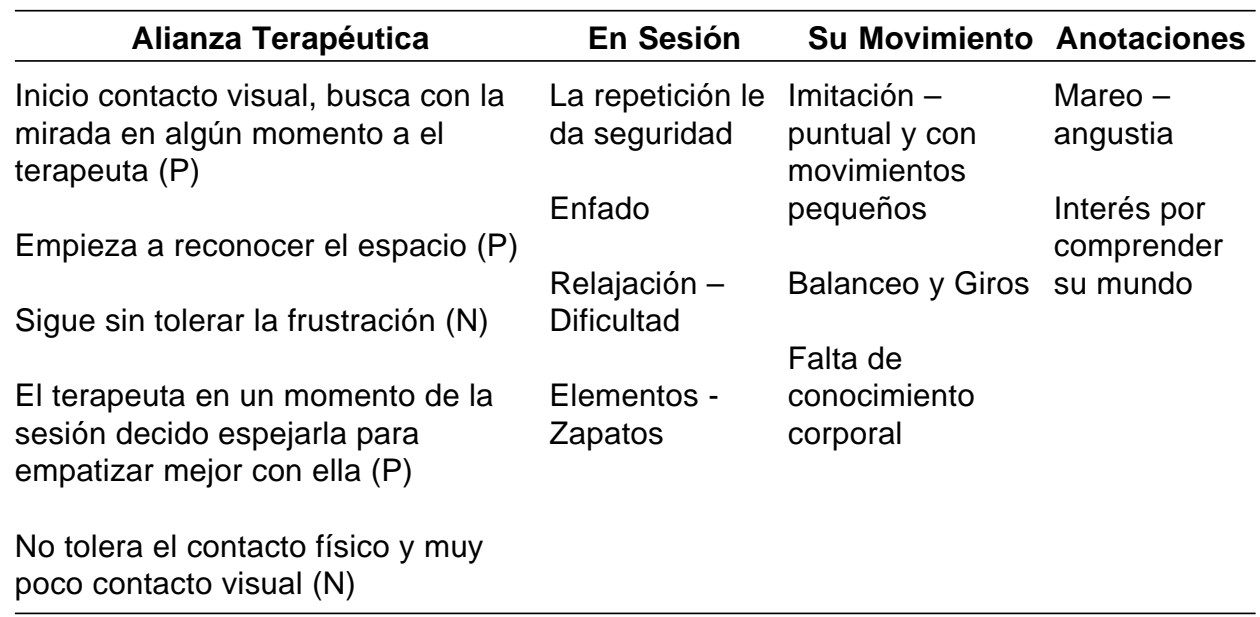

\section{Tabla 4}

Desarrollo del proceso: Sesiones 4, 5 y 6

\begin{tabular}{|c|c|c|c|}
\hline Alianza Terapéutica & En Sesión & Su Movimiento & Anotaciones \\
\hline $\begin{array}{l}\text { Integración de algunos elementos } \\
\text { del encuadre }(\mathrm{P}) \\
\text { Cuida el espacio }(\mathrm{P}) \\
\text { Se dirige a el terapeuta - pedir su } \\
\text { canción - contacto visual }(\mathrm{P}) \\
\text { Tolerancia a la frustración: acepta } \\
\text { alguna propuesta como las } \\
\text { canciones - «hay un espacio para } \\
\text { sus canciones y también hay un } \\
\text { espacio para mis canciones» - }(\mathrm{N})\end{array}$ & $\begin{array}{l}\text { Ritual - zapatos - } \\
\text { introducción del } \\
\text { calendario } \\
\text { Acoge mejor mis } \\
\text { propuestas - falta } \\
\text { atención y } \\
\text { motivación } \\
\text { Despedida - } \\
\text { «vamos» }\end{array}$ & $\begin{array}{l}\text { Giros - propuesta de } \\
\text { combinar. } \\
\text { Flujo contenido - } \\
\text { rigidez, respiración, } \\
\text { tensión cuello. } \\
\text { Espacio Indirecto: } \\
\text { mirada perdida - mira } \\
\text { mano izquierda }\end{array}$ & $\begin{array}{l}\text { Ternura - ganas } \\
\text { de protegerla } \\
\text { Objetivos } \\
\text { pequeños } \\
\text { importancia } \\
\text { vínculo } \\
\text { terapéutico } \\
\text { Marcar límites }\end{array}$ \\
\hline
\end{tabular}


Tabla 5

Desarrollo del proceso: Sesiones 7 y 8

\begin{tabular}{|c|c|c|c|}
\hline Alianza Terapéutica & En Sesión & Su Movimiento & Anotaciones \\
\hline $\begin{array}{l}\text { Recibe mejor los } \\
\text { limites y la negación } \\
\text { de sus demandas }(\mathrm{P}) \\
\text { Diálogo breve en } \\
\text { movimiento }(\mathrm{P}) \\
\text { Presente y } \\
\text { colaboradora }(\mathrm{P}) \\
\text { Tolera el contacto con } \\
\text { el propio cuerpo }(\mathrm{P})\end{array}$ & $\begin{array}{l}\text { Estructura: zapatos - } \\
\text { sentarnos - calendario } \\
\text { Pide su canción } \\
\text { Calentamiento - sigue } \\
\text { la propuesta } \\
\text { Percusión - pregunta } \\
\text { respuesta } \\
\text { Marcha rítmica - } \\
\text { explorar el espacio } \\
\text { Masaje pies }\end{array}$ & $\begin{array}{l}\text { Balanceo - Giros: } \\
\text { menos rigidez } \\
\text { Busca el contacto } \\
\text { visual y la } \\
\text { comunicación con el } \\
\text { otro } \\
\text { Combinación de } \\
\text { Espacio Directo } \\
\text { (marcha rítmica) e } \\
\text { Indirecto (giros) }\end{array}$ & $\begin{array}{l}\text { Repetición - } \\
\text { registros de } \\
\text { movimiento muy } \\
\text { reducido } \\
\text { Sentía que en } \\
\text { algunos } \\
\text { momentos me } \\
\text { incluía } \\
\text { Masaje pies - } \\
\text { indicador } \\
\text { positivo de } \\
\text { contacto con ella } \\
\text { misma }\end{array}$ \\
\hline
\end{tabular}

\section{Tabla 6}

Desarrollo del proceso: Sesiones 9, 10 y 11

\begin{tabular}{|c|c|c|c|}
\hline Alianza Terapéutica & En Sesión & Su Movimiento & Anotaciones \\
\hline $\begin{array}{l}\text { Integración elementos } \\
\text { del encuadre }(P) \\
\text { Cuida el espacio }(P) \\
\text { Proximidad }(P) \\
\text { Confianza }(P) \\
\text { Tolera mejor la } \\
\text { frustración }(P)\end{array}$ & $\begin{array}{l}\text { Iniciativa -"yo sola" } \\
\text { Inicia calentamiento: } \\
\text { "pam pam" } \\
\text { Propone movimientos } \\
\text { - "como yo" - coge } \\
\text { mi mano: } \\
\text { “ ¿bailamos?" } \\
\text { Acepta propuestas } \\
\text { Bailamos juntas } \\
\text { Pide masaje pies - } \\
\text { Contacto corporal }\end{array}$ & $\begin{array}{l}\text { Balanceo aceptando } \\
\text { pequeñas } \\
\text { modificaciones } \\
\text { Tensamos - } \\
\text { Relajamos } \\
\text { Destaca el peso firme } \\
\text { Pañuelos: Flujo más } \\
\text { libre y un tiempo más } \\
\text { sostenido } \\
\text { Ampliación registro } \\
\text { movimiento - } \\
\text { Iniciar/Parar }\end{array}$ & $\begin{array}{l}\text { Antes de salir coge } \\
\text { mi barbilla y me da } \\
\text { un beso, sale } \\
\text { rápidamente de la } \\
\text { sala } \\
\text { Está más presente en } \\
\text { el espacio - más } \\
\text { abierta al exterior } \\
\text { Juntas compartiendo } \\
\text { algo más que el } \\
\text { mismo espacio }\end{array}$ \\
\hline
\end{tabular}




\section{Tabla 7}

Desarrollo del proceso: Sesión 12

\begin{tabular}{|c|c|c|c|}
\hline Alianza Terapéutica & En Sesión & Su Movimiento & Anotaciones \\
\hline $\begin{array}{l}\text { Falta de tolerancia a la } \\
\text { frustración }(\mathrm{N})\end{array}$ & $\begin{array}{l}\text { Calendario: Rompe } \\
\text { los colores }\end{array}$ & $\begin{array}{l}\text { Mirada al suelo } \\
\text { Giros - Balanceo }\end{array}$ & $\begin{array}{l}\text { El incidente de los } \\
\text { colores la ayuda a } \\
\text { conectar con la }\end{array}$ \\
\hline $\begin{array}{l}\text { Proximidad: Inicia y } \\
\text { tolera }(P)\end{array}$ & $\begin{array}{l}\text { No acepta ninguna } \\
\text { propuesta }\end{array}$ & $\begin{array}{l}\text { Check - Out: Busca } \\
\text { contacto visual, }\end{array}$ & discusión con $X$ \\
\hline $\begin{array}{l}\text { Dificultad en } \\
\text { resolución de } \\
\text { problemas (N) }\end{array}$ & $\begin{array}{l}\text { Masaje pies: } \\
\text { Verbalización - "A se } \\
\text { enfadó conmigo , no } \\
\text { me gusta" }\end{array}$ & $\begin{array}{l}\text { cuerpo menos rígido: } \\
\text { flujo libre y peso } \\
\text { ligero }\end{array}$ & \\
\hline $\begin{array}{l}\text { Capacidad para } \\
\text { relacionar lo que pasa } \\
\text { en sesión con otros } \\
\text { momentos de su vida } \\
\text { (P) }\end{array}$ & & & \\
\hline
\end{tabular}

\section{Tabla 8}

Desarrollo del proceso: Sesiones 13 y 14

\begin{tabular}{|c|c|c|c|}
\hline Alianza Terapéutica & En Sesión & Su Movimiento & Anotaciones \\
\hline $\begin{array}{l}\text { Toma iniciativa }(P) \\
\text { Capacidad de tolerar } \\
\text { cierto grado de } \\
\text { frustración }(P) \\
\text { Busca proximidad }(P) \\
\text { Muestra confianza }(P)\end{array}$ & $\begin{array}{l}\text { Calendario - Tira los } \\
\text { colores y los recoge } \\
\text { No acepta propuestas } \\
\text { Se sienta en el suelo - } \\
\text { masaje pies y me } \\
\text { explica que ha } \\
\text { dormido poco } \\
\text { Liderazgo - imitación } \\
\text { Control Impulsos - } \\
\text { caja china }\end{array}$ & $\begin{array}{l}\text { Propone } \\
\text { movimientos } \\
\text { dentro de su } \\
\text { registro habitual } \\
\text { pero puede } \\
\text { experimentar } \\
\text { nuevos } \\
\text { movimientos con } \\
\text { mis propuestas }\end{array}$ & $\begin{array}{l}\text { Reflejar - Giros (me } \\
\text { hacen sentir perdida) } \\
\text { Me siento satisfecha } \\
\text { porque la estructura de } \\
\text { las sesiones están } \\
\text { dando a Lucía más } \\
\text { seguridad de sentirse } \\
\text { capaz y tomar iniciativa } \\
\text { "yo sola" } \\
\text { Pequeños cambios }\end{array}$ \\
\hline
\end{tabular}

\section{Tabla 9}

Desarrollo del proceso: Sesión 15

\begin{tabular}{llll}
\hline \multicolumn{1}{c}{ Alianza Terapéutica } & \multicolumn{1}{c}{ En Sesión } & \multicolumn{1}{c}{ Su Movimiento } & \multicolumn{1}{c}{ Anotaciones } \\
\hline $\begin{array}{l}\text { No busca el contacto } \\
\text { (N) }\end{array}$ & $\begin{array}{l}\text { Calendario - } \\
\text { dedicamos tiempo }\end{array}$ & Giros y Balanceo & $\begin{array}{l}\text { Frustración - } \\
\text { conecto con las } \\
\text { primeras }\end{array}$ \\
$\begin{array}{l}\text { No escucha ni acepta } \\
\text { ninguna propuesta (N) }\end{array}$ & $\begin{array}{l}\text { Solo quiere dar } \\
\text { vueltas }\end{array}$ & $\begin{array}{l}\text { corporal: flujo } \\
\text { contenido y peso firme }\end{array}$ & $\begin{array}{l}\text { sesiones con } \\
\text { Diáa }\end{array}$ \\
& Dialogo - percusión & \\
& Integramos la \\
& percusión a sus giros & \\
\hline
\end{tabular}


Tabla 10

Desarrollo del proceso y preparación para el cierre: Sesión 16 -17 y 18

\begin{tabular}{|c|c|c|c|}
\hline $\begin{array}{c}\text { Alianza } \\
\text { Terapéutica }\end{array}$ & En Sesión & Su Movimiento & Anotaciones \\
\hline $\begin{array}{l}\text { Integración del } \\
\text { encuadre }(P)\end{array}$ & $\begin{array}{l}\text { Preparamos juntas la sala - } \\
\text { Ritual }\end{array}$ & $\begin{array}{l}\text { Sobre su eje vertical: } \\
\text { centro corporal, más } \\
\text { abierta al espacio }\end{array}$ & $\begin{array}{l}\text { Satisfacción } \\
\text { por poder } \\
\text { desarrollar }\end{array}$ \\
\hline $\begin{array}{l}\text { Confianza y } \\
\text { aceptación (P) }\end{array}$ & $\begin{array}{l}\text { Calendario - Caen colores "que } \\
\text { bien, venga va" (sonríe) - Me } \\
\text { hace una caricia }\end{array}$ & $\begin{array}{l}\text { Cuerpo menos rígido - } \\
\text { más relajado }\end{array}$ & $\begin{array}{l}\text { una propuesta } \\
\text { Siento otra }\end{array}$ \\
\hline $\begin{array}{l}\text { Capacidad de } \\
\text { vincularse }(P) \\
\text { Proximidad }(P)\end{array}$ & $\begin{array}{l}\text { Calentamiento compartido: } \\
\text { esquema corporal / colocación } \\
\text { del cuerpo }\end{array}$ & $\begin{array}{l}\text { Aparece la mano } \\
\text { izquierda en los giros } \\
\text { pero en poco momentos }\end{array}$ & $\begin{array}{l}\text { energía en } \\
\text { nuestro } \\
\text { vinculo }\end{array}$ \\
\hline $\begin{array}{l}\text { Inicia interacción } \\
(\mathrm{P})\end{array}$ & $\begin{array}{l}\text { Marcha rítmica - Nuestro } \\
\text { eje/centro }\end{array}$ & Giros - más abiertos & $\begin{array}{l}\text { Tristeza por el } \\
\text { cierre }\end{array}$ \\
\hline $\begin{array}{l}\text { Aumento } \\
\text { autonomía }(P)\end{array}$ & $\begin{array}{l}\text { Dialogo instrumentos percusión } \\
\text { "yo sola" y percusión corporal }\end{array}$ & Flujo menos contenido & $\begin{array}{l}\text { Incertidumbre } \\
\text { respecto la } \\
\text { última sesión }\end{array}$ \\
\hline $\begin{array}{l}\text { Aprendizaje - } \\
\text { resolver } \\
\text { algunas } \\
\text { situaciones }(P)\end{array}$ & $\begin{array}{l}\text { Masaje pies y Canción } \\
\text { despedida }\end{array}$ & & \\
\hline
\end{tabular}

Tabla 11

Cierre del proceso: Sesión 19

\begin{tabular}{|c|c|c|c|}
\hline Alianza Terapéutica & En Sesión & Su Movimiento & Anotaciones \\
\hline $\begin{array}{l}\text { Integración elementos } \\
\text { encuadre }(P)\end{array}$ & $\begin{array}{l}\text { Preparamos juntas la } \\
\text { sala - Rituales - }\end{array}$ & $\begin{array}{l}\text { Cuerpo menos rígido, } \\
\text { cuello menos inclinado. }\end{array}$ & $\begin{array}{l}\text { Dibujo: Algo } \\
\text { físico para } \\
\text { ayudarle a }\end{array}$ \\
\hline $\begin{array}{l}\text { Capacidad de vincularse } \\
\text { (P) }\end{array}$ & $\begin{array}{l}\text { Verbalizo que es la } \\
\text { última sesión }\end{array}$ & Respiración más amplia & cerrar \\
\hline $\begin{array}{l}\text { Capacidad de tolerar la } \\
\text { frustración }(P)\end{array}$ & $\begin{array}{l}\text { Dibujamos nuestras } \\
\text { manos }\end{array}$ & $\begin{array}{l}\text { Se observa } \\
\text { combinación: flujo } \\
\text { libre y contenido }\end{array}$ & $\begin{array}{l}\text { Los pequeños } \\
\text { cambios }\end{array}$ \\
\hline \multirow[t]{2}{*}{$\begin{array}{l}\text { Proximidad - Inicia } \\
\text { contacto }(P)\end{array}$} & $\begin{array}{l}\text { Despedida: "pequeña } \\
\text { fiesta" }\end{array}$ & $\begin{array}{l}\text { Kinesfera personal y } \\
\text { general ampliada }\end{array}$ & \\
\hline & $\begin{array}{l}\text { Masaje pies y canción } \\
\text { despedida }\end{array}$ & Giros & \\
\hline
\end{tabular}




\section{Agradecimientos}

A la Fundació Blanquerna (FPCEE Blanquerna de la Universitat Ramon Lull) por la beca de investigación concedida. A la formación de Master de DMT (Universitat Autònoma de Barcelona). Y a Lucía.

\section{Referencias bibliográficas}

AAIDD (2010). Discapacidad Intelectual. Definición, clasificación y sistemas de apoyo. Madrid: Alianza Editorial.

American Psychiatric Association. (2013). Diagnostic and statistical manual of mental disorders (Vol. 5). Washington: American Psychiatric Publishing.

Andrade, N. (2005). La alianza terapéutica. Clínica y Salud. 16(1), 9-29.

Aron L. (1999) Afterword. En: Mitchell S. Aron L. Relational Psychoanalysis: The Emergence of a Tradition. The Analytic Press, New Jersey.

Arregui, I. (2010). La técnica del psicodrama utilizada en adolescentes, como medio para prevenir el maltrato en el aula de clases: trabajo de campo. Colegio Nacional Cumbayá.

Asociación Médica Mundial. (1964) Principios éticos para la investigación médica con sujetos humanos. XVII Asamblea Mundial de la Asociación Médica Mundial. Helsinki, Finlandia.

Ballesta, A. M., Vizcaíno, O., y Mesas, E. C. (2011). El Arte como un lenguaje posible en las personas con capacidades diversas. Arte y politicas de identidad, 4, 137-152.

Barnet-López, S., Pérez-Testor, S., Cabedo-Sanromà, J., Gozzoli, C., Oviedo, G. R., y Guerra-Balic, M. (2015). Developmental Items of Human Figure Drawing: Dance/Movement Therapy for Adults with Intellectual Disabilities.American Journal of Dance Therapy, 37(2), 135-149. doi: 10.1007/s10465-015-9201-1.

Beck, A.T., Rush, A.J., Shaw, B.B, y Emery, G. (1983). Terapia cognitiva de la depresión. Bilbao: Editorial DDB.

Bordin, E. S. (1979). The generalizability of the psychoanalytic concept of the working alliance. Psychotherapy: Theory, research and practice, 16(3), 252.

Bordin, E. S. (1994). Theory and research on the therapeutic working alliance: New directions. The working alliance: Theory, research and practice, 13-37.

Borja, V., y Solís P. (2012). Taller de música y danza: Expresión Corporal en un centro de apoyo a la integración de personas con discapacidad intelectual. EmásF: revista digital de educación física, 14, 52-59.

Botella, L., y Corbella, S. (2011). Alianza terapéutica evaluada por el paciente y mejora sintomática a lo largo del proceso terapéutico. Boletín de psicología, (101), 21-34.

Chace, M. (1953). Dance as an adjunctive therapy with hospitalized mental patients. Bulletin of the Menninger Clinic, 17, 219-225.

Corbella, S., Balmaña, N., Fernández-Álvarez, H., Saúl, L. A., Botella, L., y García, F. (2009). estilo personal del terapeuta. Revista Argentina de Clínica Psicológica, 18, 125-133.

Corbella, S., y Botella, L. (2003). La alianza terapéutica: historia, investigación y evaluación. Anales de psicología, 19(2), 205-221.

Etchevers, M., Garay, C. J., Korman, G. P., González, M. M., y Simkin, H. (2012). Principales desarrollos y enfoques sobre alianza y relación terapéutica. Anuario de investigaciones, 19(1), 225-232.

Fischman, D. (2005). Danza Movimiento Terapia. Encarnar, enraizar y empatizar. Construyendo los mundos en que vivimos. Congreso de Artes del Movimiento. IUNA: Buenos Aires.

Gibbs, G.R. (2007). Analysing qualitative data. London : Sage Publications.

González, N.A. (2005). La alianza terapéutica. Clínica y Salud, 16(1), 9-29.

Greenson, R. (1967). Technique and practice of psychoanalysis. New York: International.

Horvarth, A.O. (1981). An exploratory study of the working Alliance: Its measurement and reationship to therapy outcome. U. British Columbia, Vancouver, Canadá.

Horvath, A.O., y Bedi, R.P. (2002). The alliance. En J. C. Norcross (Ed.), Psychotherapy relationships that work. 37-70.

Horvath, A.O. y Greenberg, L.S. (1989). Development and validation of the Working Alliance Inventory. Journal of Counseling Psychology, 36, 223-233. 
Hurley, A., Tomasulo, D. y Fadt, A. (1998). Individual and group psychotherapy: Approaches for persons with mental retardation and developmental disabilities. Journal of Developmental and Physical Disabilities, 10 (4), 365-386.

Jané, M.C. (2004). Diagnòstic dual en la deficiencia mental. Suports, 8(1), 42-58.

Koch, S., Kunz, T., Lykou, S., y Cruz, R. (2014). Effects of dance movement therapy and dance on health-related psychological outcomes: A meta-analysis. The Arts in Psychotherapy, 41(1), 46-64. doi:10.1016/ j.aip.2013.10.004

Laban, R. (1987). El Dominio del Movimiento. Madrid: Ed. Fundamentos.

Levy F. (1992). Dance Movement Therapy a Healing Art. New York, Ed. National Dance Association, American Alliance for Health, Physical Education, Recreation and Dance.

Levy, F. (1988). Dance Movement Therapy: A Healin Art. Virginia: The American Aliance for Health, Physical Education, Recreation, and Dance.

Luck, L., Jackson, D., y Usher, K. (2006). Case study: a bridge across the paradigms. Nursing inquiry, 13(2), 103109.

MacDonald, J. (1992). Dance? Of cours I can! En H. Payne (Ed.) Dance Movement Therapy: Theory and Practice. Routledge: London.

Miles, M.B., y Huberman, A.M. (1994). Qualitative data analysis: An expanded sourcebook. 1994. Beverly Hills: Sage Publications.

Panhofer, H. (2005). El cuerpo en psicoterapia. Teoría y práctica de la Danza Movimiento Terapia. Barcelona: Gedisa Editorial.

Peschken, W.E. y Johnson, M.E. (1997). Therapist and client trust in the therapist and client trust in the therapeutic relationship. Psychotherapy Research, 7 (4), 439-447.

Rodas, J. L. C. (2015). Danza Terapia: alternativa de bienestar en Colombia. Artes la Revista, 7(14), 61-68.

Rogers, C y Resenberg, C. (1981). La persona como centro. Barcelona: Herder.

Ruiz, J.I. (1996). Metodología de investigación cualitativa. Bilbao: Universidad de Deusto.

Sassenfeld, A. (2008) Lenguaje corporal e intencionalidad relacional. Gaceta de Psiquiatría Universitaria. 4, 1.

Starman, A. B. (2013). The case study as a type of qualitative research. Journal of Contemporary Educational Studies/Sodobna Pedagogika, 64(1), 28-43.

Sterba, R. (1934). The fate of the ego in analytic therapy. International Journal of Psychoanalysis, 115, 117-126.

Stern, D.N., Sander, L.W., Nahum, J.P., Harrison, A.M., Lyons-Ruth, K., Morgan, A.C., Bruschweilerstern, N. y Tronick, E.Z. (1998). Mecanismos no interpretati-vos en terapia psicoanalítica. El “Algo más” la interpretación, traducción al castellano del artículo: Non-interpretive mechanisms in psychoanalytic thera-py: the "Something more" than interpretation. International Journal of Psychoanalysis, 79, 903-921. University Press.

Vella, G., y Torres, E. (2012). Desarrollo de un instrumento de observación en danza movimiento terapia (DMT). Papeles del Psicólogo, 33(1), 3-13.

Watson, J.C., y Geller, S.M.(2005). The relation among the relationship conditions, working alliance, and outcome in both process-experiential and cognitive-behavioral psychotherapy. Psychotherapy Research, 15(1-2), 25-33.

Winkler, M.I., Cáceres, C., Fernández, I., y Sanhueza, J. (1989). Factores inespecíficos de la psitoterapia y efectividad del proceso terapéutico: una sistematización. Revista Terapia Psicológica, 8(11), 34-40. 